$15^{\text {th }}$ International Conference on

AEROSPACE SCIENCES \& AVIATION TECHNOLOGY,

$\boldsymbol{A S A T}$ - 15 - May 28 - 30, 2013, Email: asat@mtc.edu.eg,

Military Technical College, Kobry Elkobbah, Cairo, Egypt,

Tel: +(202) 24025292 -24036138, Fax: +(202) 22621908

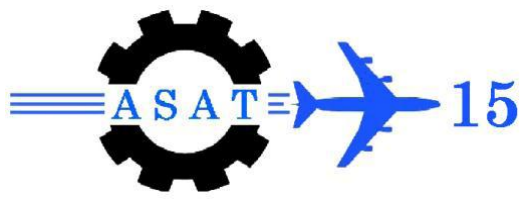

\title{
Vehicle's Dynamics Performance in Response to Accelerometer Positioning
}

\author{
M. S. Mohamed*, A. A. El-Ramlawy ${ }^{\dagger}$, G. A. El-Sheikh ${ }^{\ddagger}$ and A. M. Bayoumy ${ }^{\S}$
}

\begin{abstract}
In an airplane of tail-controlled aerodynamic missiles, when the control surfaces are deflected to provide an elevator angle with trailing edge upward to produce a positive normal acceleration, this creates a downward increment of force on the tail. The result is that the missile c.g. may drop momentarily during the pitch-up, so the normal acceleration may briefly become negative before it builds up positively. If the accelerometer is located at missile c.g., it will sense a negative normal acceleration and the senses a positive normal acceleration in a manner similar to non-minimum phase system. If the accelerometer is located ahead of the c.g., the normal acceleration measured will change and this depends on the pitch acceleration about the c.g., so only a positive normal acceleration may be sensed. This paper represents the physical explanation for the non-minimum phase behavior in tailcontrolled aerodynamic missiles, its source, disadvantages and solution for this problem and finally the optimum location for accelerometer in the vehicle.
\end{abstract}

Keywords: Missile flight control, accelerometers, non-minimum phase system

\section{Nomenclature}

\begin{tabular}{|c|c|c|c|}
\hline $\mathrm{A}, \mathrm{B}, \mathrm{C}$ & State-space matrices & Q & Dynamic pressure \\
\hline$a_{n}$ & Normal acceleration at accelerometer position & $\mathrm{q}_{\mathrm{c}}$ & Commanded pitch rate \\
\hline$a_{\mathrm{nc}}$ & Commanded normal acceleration & S & Laplace operator \\
\hline$a_{n, c g}$ & Normal acceleration at c.g position & $\mathrm{t}$ & Time \\
\hline$e_{\text {an }}$ & Error in normal acceleration & $\mathrm{u}, \mathrm{v}, \mathrm{w}$ & Velocity component in body axes \\
\hline$e_{q}$ & Error in pitch rate & $\mathrm{u}_{0}$ & Undisturbed longitudinal velocity \\
\hline $\mathrm{G}_{\mathrm{c}}$ & Compensator transfer function & $\mathrm{u}_{\eta}$ & Control signal \\
\hline $\mathrm{G}_{\mathrm{fp}}$ & Forward path transfer function & $\mathrm{X}_{\mathrm{u}}$ & Axial force due to longitudinal velocity \\
\hline $\mathrm{K}_{\mathrm{acc}}$ & Accelerometer gain & $\mathrm{X}_{\mathrm{a}}$ & Accelerometer position from missile nose \\
\hline $\mathrm{K}_{\mathrm{q}}$ & Pitch rate gain & $\mathrm{Y}_{\mathrm{r}}$ & Side force due to yaw rate \\
\hline $\mathrm{L}_{\mathrm{p}}$ & Rolling moment due to roll rate & $\mathrm{Y}_{\mathrm{v}}$ & Side force due to side velocity \\
\hline $\mathrm{L}_{\xi}$ & Rolling moment due to aileron angle & $\mathrm{Y}_{\zeta}$ & Side force due to rudder angle \\
\hline $1_{\mathrm{p}}$ & Accelerometer position in front of c.g. & $\mathrm{Z}_{\mathrm{q}}$ & Normal force due to pitch rate \\
\hline M & $\begin{array}{l}\text { Pitching moment, Resultant of external } \\
\text { moments w.r.t. body axes }\end{array}$ & $\mathrm{Z}_{\mathrm{w}}$ & Normal force due to vertical velocity \\
\hline Ma & Mach number & $\mathrm{Z}_{\alpha}$ & Normal force due to angle of attack \\
\hline $\mathrm{M}_{\mathrm{q}}$ & Pitching moment due to pitch rate & $\mathrm{Z}_{\eta}$ & Normal force due to elevator angle \\
\hline $\mathrm{M}_{\mathrm{w}}$ & Pitching moment due to vertical velocity & $\alpha$ & Angle of attack \\
\hline $\mathrm{M}_{\alpha}$ & Pitching moment due to angle of attack & $\beta$ & Side slip angle \\
\hline
\end{tabular}

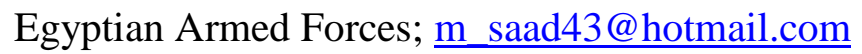

$\dagger \quad$ Egyptian Armed Forces; abaset92@yahoo.com

$\$ \quad$ Prof. Pyramids Higher Institute for Engineering and Technology, gaelsheikh@gmail.com

$\S \quad$ Egyptian Armed Forces; ambayoumy@gmail.com
} 
$\mathrm{M}_{\eta} \quad$ Pitching moment due to elevator angle

$\mathrm{N} \quad$ Transfer function numerator

$\mathrm{N}_{\mathrm{r}} \quad$ Yawing moment due to yaw rate

$\mathrm{N}_{\mathrm{v}} \quad$ Yawing moment due to side velocity

$\mathrm{N}_{\zeta} \quad$ Yawing moment due to rudder angle

$\mathrm{p}, \mathrm{q}, \mathrm{r} \quad$ Roll, pitch and yaw rates $\zeta \quad$ Rudder angle

$\zeta_{\mathrm{sp}} \quad$ Short period mode damping ratio

$\eta \quad$ Elevator angle

$\xi \quad$ Aileron angle

$\omega_{\text {sp }} \quad$ Short period mode natural frequency

\section{Introduction}

The physical explanation for the non-minimum phase behavior is that when the control fins are deflected to provide an elevator angle with trailing edge upward to produce a positive normal acceleration $a_{n}$, this creates a downward increment of force on the tail. The result is that the missile c.g. may drop momentarily during the pitch-up, so the normal acceleration may briefly become negative before it builds up positively and at this small time the missile performs general plane motion. During this time, if the accelerometer is translated ahead of the c.g., the normal acceleration measured will change and this depends on the pitch acceleration about the c.g., till a point on the missile the accelerometer senses zero acceleration and only rolling motion is sensed. This point is called "instantaneous center of rotation". If the accelerometer is place ahead of this point, so only a positive normal acceleration will be sensed [1].

The non-minimum phase system is undesirable from a feedback point of view as this zero cannot be removed from a system through inverse compensation (pole-zero cancelation) because it is never known exactly due to change of missile parameters during flight. The compensation to remove it would be unstable, and if there was any error in the zero location, the zero would not be cancelled and the compensator and would introduce instability [1], [2].

In this paper, investigation for non-minimum phase behavior will be performed representing its reason and its solution and the derivation of position of "instantaneous center of rotation" along the flight time including the design of normal acceleration autopilot.

\section{Missile Model}

Equations of motion from [1] and [4], aerodynamic coefficients are calculated from [5] and as represented in [6].

\section{Linearization of Missile Model}

The linear equations needed for control system design will be derived using the small perturbation method from the nonlinear model. In [7], a complete linearization for force and moment equations in the state model is presented for design of model-predictive controllers. The six equations of motion can be written as: 


$$
\left[\begin{array}{c}
\Delta \dot{u} \\
\Delta \dot{v} \\
\Delta \dot{w} \\
\Delta \dot{p} \\
\Delta \dot{q} \\
\Delta \dot{r}
\end{array}\right]=\left[\begin{array}{cccccc}
X_{u} & 0 & 0 & 0 & 0 & 0 \\
0 & Y_{v} & 0 & 0 & 0 & \left(Y_{r}-u_{0}\right) \\
0 & 0 & Z_{w} & 0 & \left(u_{0}+Z_{q}\right) & 0 \\
0 & 0 & 0 & L_{p} & 0 & 0 \\
0 & 0 & M_{w} & 0 & M_{q} & 0 \\
0 & N_{v} & 0 & 0 & 0 & N_{r}
\end{array}\right]\left[\begin{array}{l}
u \\
v \\
w \\
p \\
q \\
r
\end{array}\right]+\left[\begin{array}{ccc}
0 & 0 & 0 \\
0 & 0 & Y_{\zeta} \\
0 & Z_{\eta} & 0 \\
L_{\xi} & 0 & 0 \\
0 & M_{\eta} & 0 \\
0 & 0 & N_{\zeta}
\end{array}\right]\left[\begin{array}{l}
\xi \\
\eta \\
\zeta
\end{array}\right]
$$

\section{Choice of Trim Conditions}

In order to select the design points, the Mach number and altitude must be plotted with the flight time, or instead of them the dynamic pressure can be introduced with the change of flight time as shown in Fig. 1.

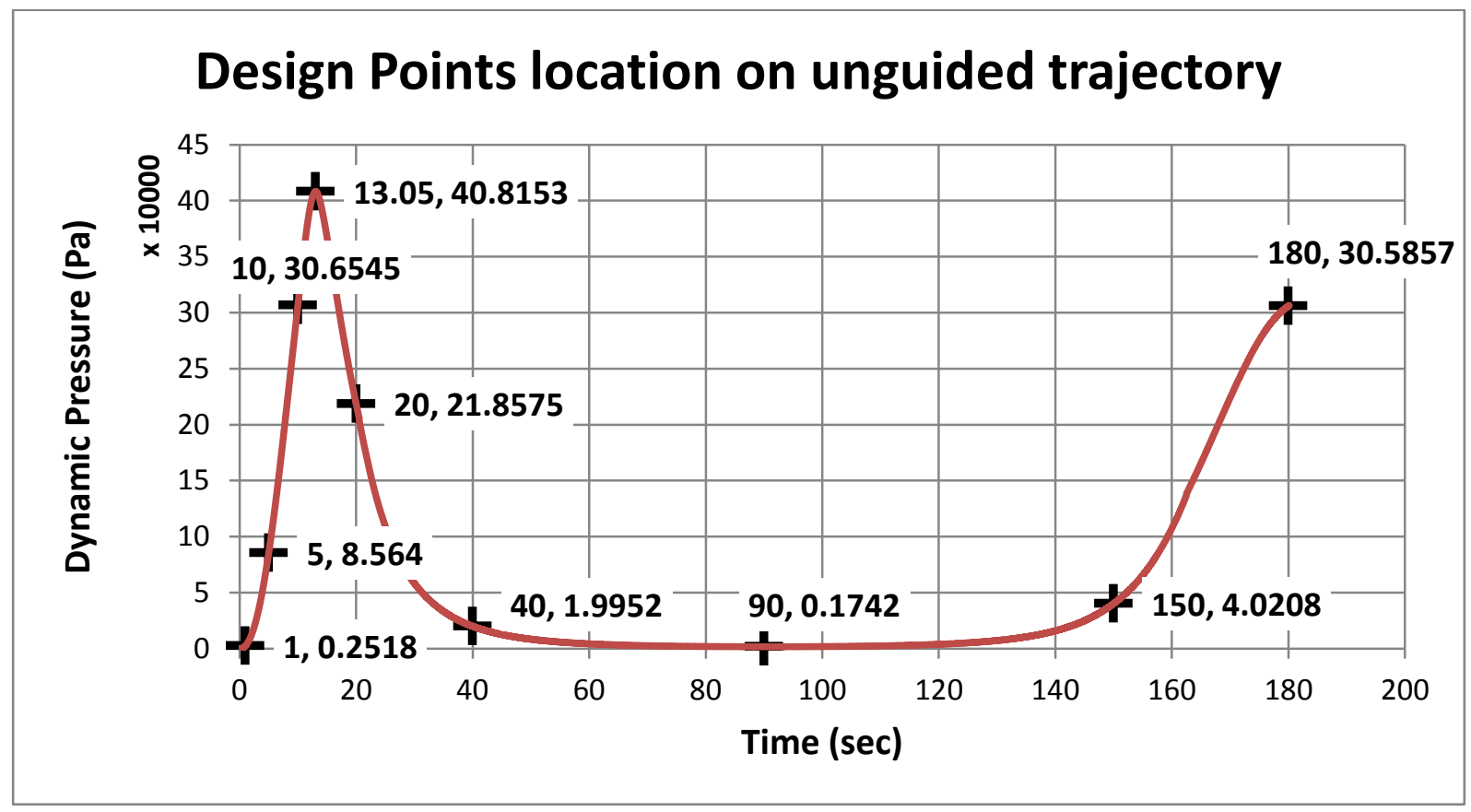

Fig. 1 Time variation of dynamic pressure

The design points must be at different dynamic pressure during the powered phase (which is from 0 to $13 \mathrm{sec}$ ) and unpowered phase (from $13 \mathrm{sec}$ till flight end) in order to avoid repeating of design points or introducing large number of design points. Due to rapid change in dynamic pressure and missile states during the powered phase, a point is selected at every $5 \mathrm{sec}$. Due to moderate change in dynamic pressure and missile parameters during the unpowered phase, it is divided into regions with mid and final-points for each region are selected. Then the set of designing points are shown in Table 1 and Fig. 1.

Table 1 Set of designing points

\begin{tabular}{c|c|c|c|c|c|c|c|c|c}
\hline \hline point & 1 & 2 & 3 & 4 & 5 & 6 & 7 & 8 & 9 \\
\hline Time [sec] & 1 & 5 & 10 & 13 & 20 & 40 & 90 & 150 & 180 \\
\hline \hline
\end{tabular}


It is necessary to select a point at which the autopilot design is carried out and to generalize the structure of the controller for the other points. This point needed to be of moderate coefficients to be near to higher dynamic pressure point and lower dynamic parameters point or in between of them. From Fig. 1, it will be acceptable if choosing point at time $(\mathrm{t}=5 \mathrm{sec})$ to be the nominal design point which has the state-space model for pitch rate and normal acceleration at missile c.g.:

$$
\begin{gathered}
{\left[\begin{array}{c}
\dot{\alpha} \\
\dot{q} \\
\dot{\eta}
\end{array}\right]=\left[\begin{array}{ccc}
-0.2253 & 0.9977 & -0.00218 \\
-20.9856 & -0.4221 & -0.47785 \\
0 & 0 & -60
\end{array}\right]\left[\begin{array}{l}
\alpha \\
q \\
\eta
\end{array}\right]+\left[\begin{array}{c}
0 \\
0 \\
60
\end{array}\right] u_{\eta}} \\
{\left[\begin{array}{c}
a_{n, c g} \\
q
\end{array}\right]=\left[\begin{array}{ccc}
8.896 & 0.0893 & 0.086 \\
0 & 1 & 0
\end{array}\right]\left[\begin{array}{l}
\alpha \\
q \\
\eta
\end{array}\right]}
\end{gathered}
$$

\section{Controller Design}

\section{Normal Acceleration Autopilot Design}

The autopilot is designed to control the normal acceleration, which its input is a desired normal acceleration $\left(\mathrm{a}_{\mathrm{nc}}[\mathrm{g}]\right)$ command sent from the guidance-loop. The complete normal acceleration control system block diagram is shown in Fig. 2, where the inner loop is the pitch damper system to ensure adequate damping ratio.

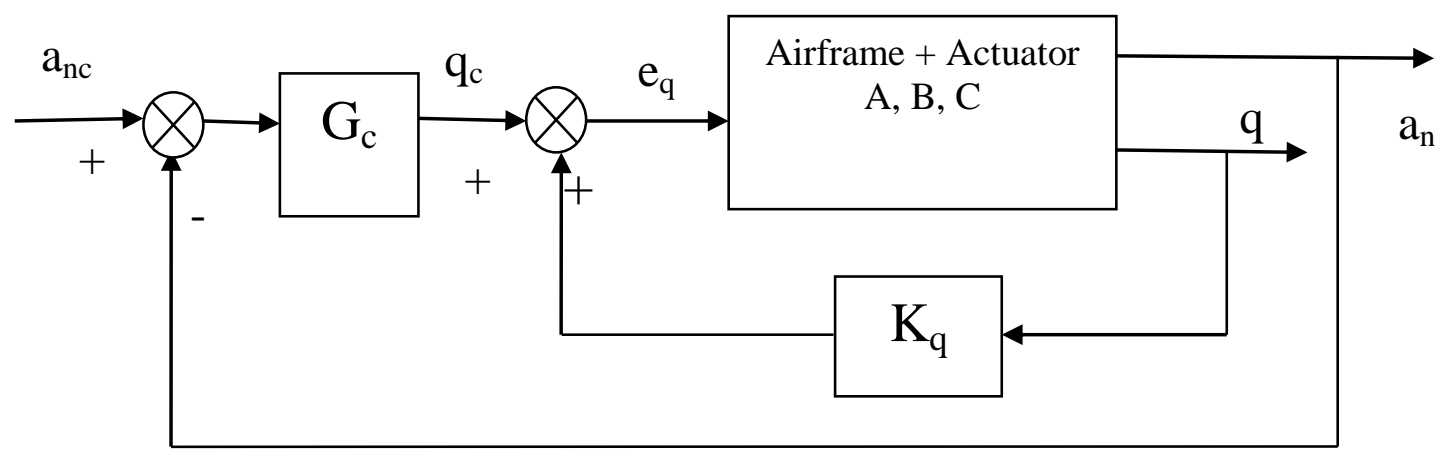

Fig. 2 Normal acceleration control system block diagram

\section{Inner Loop Design}

The block diagram of the inner-loop is shown in Fig. 3, which shows its structure with Ga is the actuator transfer function, $G_{p}$ is the airframe transfer function and $K_{q}$ is the feedback gain.

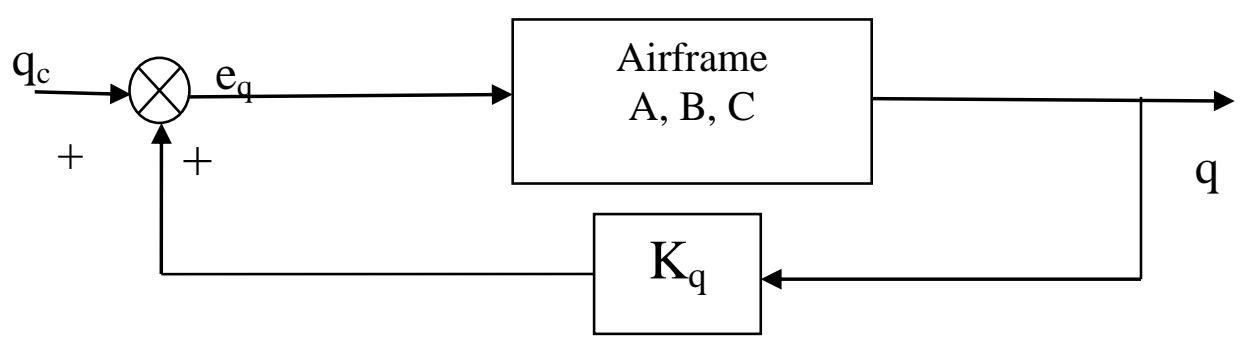

Fig. 3 Block diagram of normal acceleration inner-loop 
Introducing the airframe state space for inner loop at $(\mathrm{t}=5 \mathrm{sec})$ :

$$
\begin{gathered}
{\left[\begin{array}{c}
\dot{\alpha} \\
\dot{q} \\
\dot{\eta}
\end{array}\right]=\left[\begin{array}{ccc}
-0.2253 & 0.9977 & -0.00218 \\
-20.9856 & -0.4221 & -0.47785 \\
0 & 0 & -60
\end{array}\right]\left[\begin{array}{l}
\alpha \\
q \\
\eta
\end{array}\right]+\left[\begin{array}{l}
0 \\
0 \\
60
\end{array}\right] u_{\eta}} \\
{[q]=\left[\begin{array}{lll}
0 & 1 & 0
\end{array}\right]\left[\begin{array}{l}
\alpha \\
q \\
\eta
\end{array}\right]}
\end{gathered}
$$

The characteristic equation of the above system can be obtained from the determinant of the matrix $|\mathrm{sI}-\mathrm{A}|$ as:

$$
s^{3}+60.65 s^{2}+\left(59.88+28.67 \mathrm{~K}_{\mathrm{q}}\right) s+\left(1262+3.7147 \mathrm{~K}_{\mathrm{q}}\right)=0
$$

Rearranging this equation yields:

$$
1+\mathrm{K}_{\mathrm{q}} \frac{28.67 \mathrm{~s}+3.7147}{\mathrm{~s}^{3}+60.65 s^{2}+59.88 \mathrm{~s}+1262}=0
$$

The root locus of $1+\mathrm{K}_{\mathrm{q}} \mathrm{G}=0$ for gain $\mathrm{K}_{\mathrm{q}}>0$ is shown in Fig. 4 Fig. 4 where the poles for $\left(\mathrm{K}_{\mathrm{q}}=1\right)$ are obtained as: Short period poles: $-0.589 \pm 4.57 \mathrm{i} \rightarrow\left(\zeta_{\mathrm{sp}}=0.128, \oplus_{\mathrm{sp}}=4.61 \mathrm{rad} / \mathrm{sec}\right)$

Actuator pole: first order $-59.2 \rightarrow(\zeta=1, \omega=59.2 \mathrm{rad} / \mathrm{sec})$

The two short period complex poles $(-0.3237 \pm 4.575 \mathrm{i})$ have low damping ( $\zeta \mathrm{sp}=0.0705)$ and move on the imaginary axis due to increase of $\mathrm{K}_{\mathrm{q}}$ and then intersecting on the real axis, one of them moves to the short period zero (-0.13) and the other pole intersects with actuator pole (-60) and moves on the imaginary axis towards infinity. By increasing the value of $\mathrm{K}_{\mathrm{q}}$, the damping ratio of the short period mode increases about 0.7 where the value of $K_{q}=11.8$ at $\left(\zeta_{\text {sp }}=0.706\right)$ Fig. 4 [8].

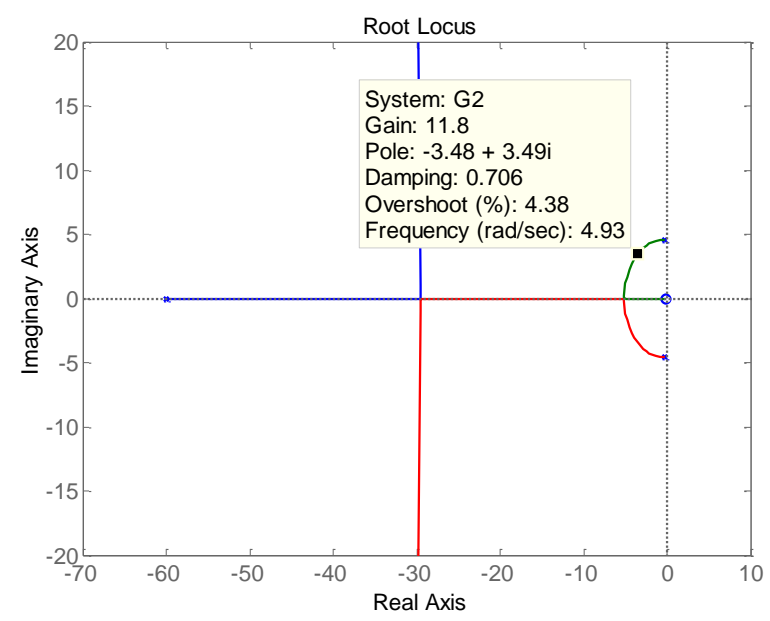

Fig. 4 Root locus of pitch damper loop 


\section{Gain Scheduling}

The nominal mathematical model, used in designing the control laws, only approximates the behavior of the physical missile, and even then at specific flight points only. Gain scheduling of $\mathrm{K}_{\mathrm{q}}$ with dynamic pressure is accepted as being appropriate for good responses across the whole flight trajectory and to ensure acceptable damping ratio. The methodology of this method is to adjust the gain multiplied $\left(\mathrm{K}_{\mathrm{q}}\right)$ in the inner loop and then scheduling the gain with the flight conditions [9]. The scheduling is shown in Table 2.

Table $2 \quad K_{\mathbf{q}}$ and $K_{\mathbf{a}}$ scheduling

\begin{tabular}{c|c|c|c|c|c|c|c|c}
\hline \hline Time & 5 & 10 & 13 & 20 & 40 & 90 & 150 & 180 \\
\hline $\mathrm{Q}[\mathrm{Pa}]$ & 85640 & 306545 & 408153 & 218575 & 19952 & 1742 & 40208 & 305857 \\
\hline $\mathrm{Kq}$ & 11.8 & 5.09 & 3.75 & 5.23 & 22.3 & 86 & 15.7 & 5.53 \\
\hline$\zeta_{\text {sp }}$ & 0.707 & 0.707 & 0.707 & 0.707 & 0.707 & 0.707 & 0.707 & 0.707 \\
\hline \hline
\end{tabular}

\section{Transfer Function Change with Accelerometer Position}

If the accelerometer is placed at missile c.g., then the normal acceleration sensed at the missile c.g. yields [1]:

$$
a_{n, c g}=\left(-Z_{\alpha} \alpha-Z_{q} q-Z_{\eta} \eta\right) / 9.81 \quad[\mathrm{~g}]
$$

The normal acceleration block diagram is shown in Fig. 5 where the state space of the outer-loop is:

$$
\begin{aligned}
& {\left[\begin{array}{c}
\dot{\alpha} \\
\dot{q} \\
\dot{\eta}
\end{array}\right]=\left[\begin{array}{ccc}
-0.2253 & 0.9977 & -0.00218 \\
-20.9856 & -0.4221 & -0.47785 \\
0 & 11.8 & -60
\end{array}\right]\left[\begin{array}{l}
\alpha \\
q \\
\eta
\end{array}\right]+\left[\begin{array}{c}
0 \\
0 \\
60
\end{array}\right] q_{c}} \\
& {\left[a_{n}\right]=\left[\begin{array}{lll}
8.896 & 0.0893 & 0.086
\end{array}\right]\left[\begin{array}{c}
\alpha \\
q \\
\eta
\end{array}\right]}
\end{aligned}
$$

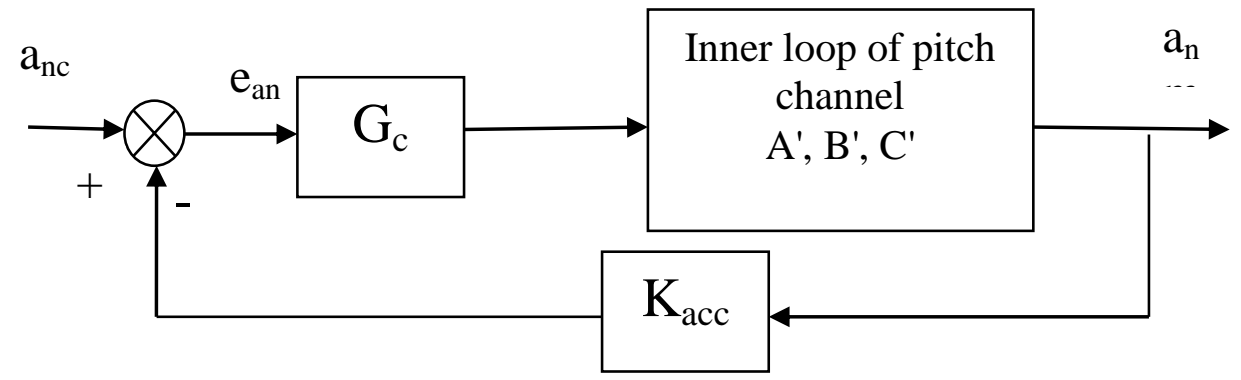

Fig. 5 Normal acceleration pitch channel block diagram 
Considering the accelerometer gain $\mathrm{K}_{\mathrm{acc}}=1$, the forward-path transfer function without the compensator is:

$$
\begin{aligned}
G_{f p} & =\frac{5.162 s^{2}-0.3825 s-146.7}{s^{3}+60.65 s^{2}+398.2 s+1306}=(5.162) \frac{(s-5.368)(s+5.294)}{(s+53.7)\left(s^{2}+6.96 s+24.32\right)} \\
& =(-2.7317) \frac{(1-0.1863 s)(1+0.189 s)}{(1+0.0186 s)\left(s^{2}+6.96 s+24.32\right)}
\end{aligned}
$$

The root locus and bode plot of the forward path transfer function is shown in Fig. 6, while the step response of closed-loop transfer function is shown in Fig. 6. The dynamics of the channel has: Zeros: $+5.368,-5.294$

Poles: Actuator pole -53.7

Short period poles $(-3.48 \pm 3.49 \mathrm{i})$

The responses clarify that there are two problems; the first one is that the response of the normal acceleration is reversed to the negative effect as shown in Fig. 6 and this problem can be solved by adding an inverter in the forward path. The second problem appears in the vicinity of positive zero $(+5.368)$, which in turn results in a non-minimum phase system behavior in the step response of the normal acceleration as shown in Fig. 7.
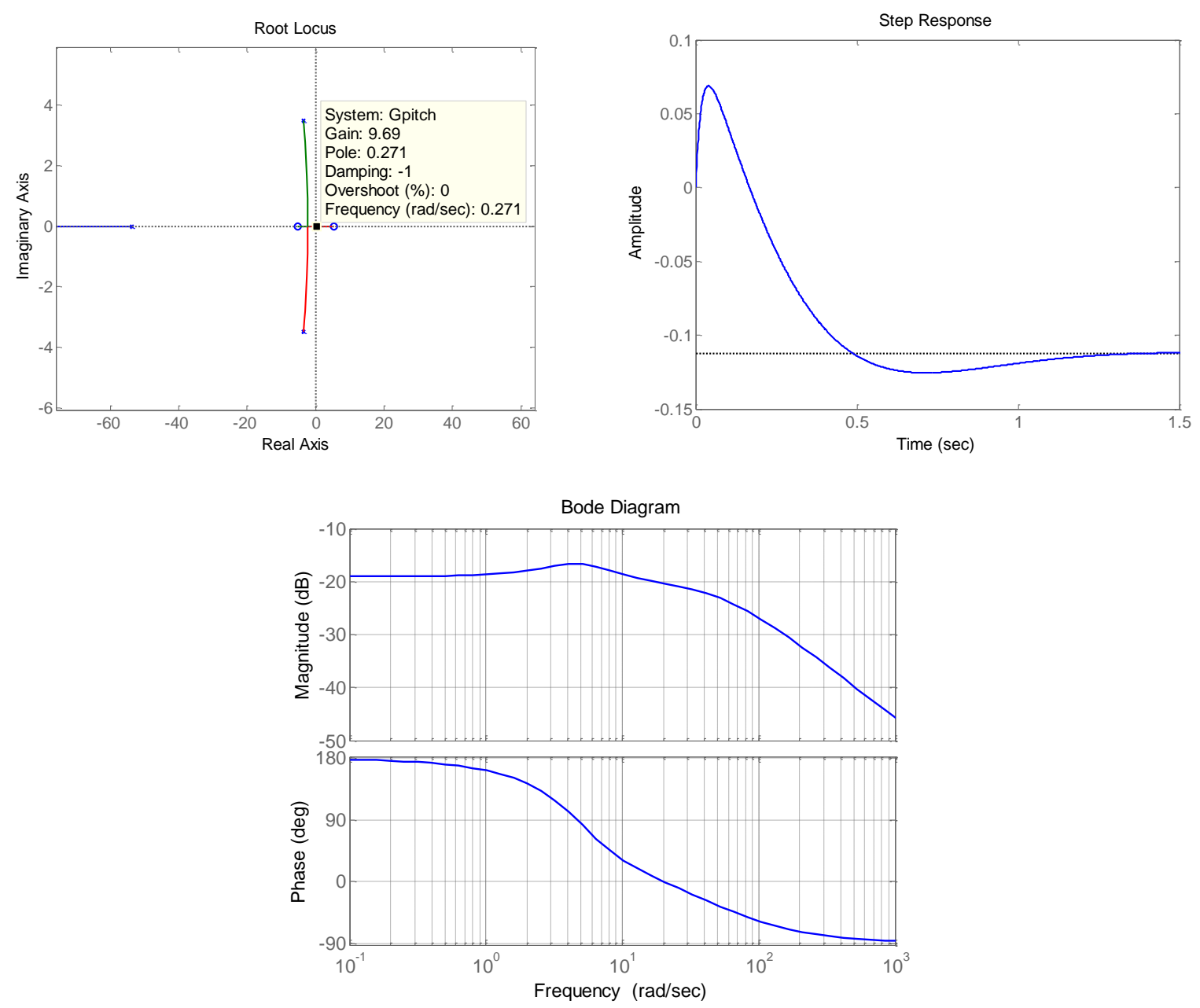

Fig. 6 Root locus, bode plot and step response of normal acceleration 


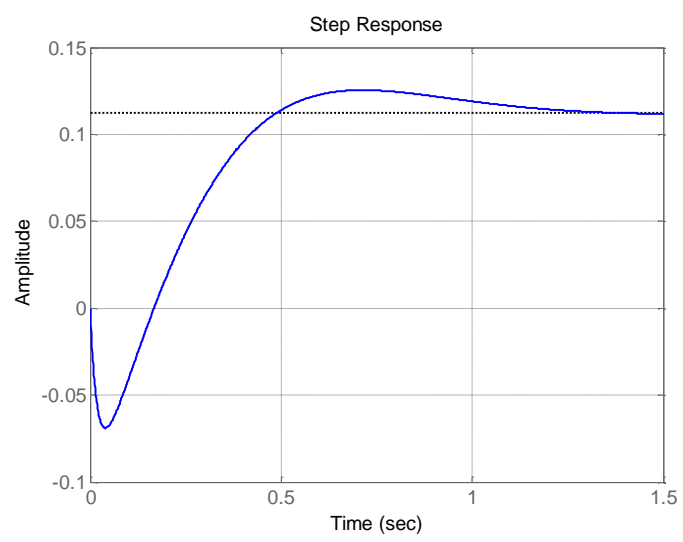

\section{Fig. 7 Step response of normal acceleration before compensation}

From Fig. 6, the root locus shows that one of the open-loop short period poles moves towards the zero and thus the non-minimum phase system goes unstable at gain $(K=9.69)$.

Actually in designing the normal acceleration autopilot, the steady-state error must equal zero. As the system is of type zero, an integrator in the forward path is required and adding this integrator pole in the root locus in Fig. 6 will cause moving of this pole into right hand plane to nonminimum phase zero and results in system instability. So, this zero must be eliminated not only to remove the non-minimum phase behavior of the normal acceleration response, but also to maintain system stability.

If the accelerometer is placed at a distance $l_{p}$ ahead of missile c.g., then the normal acceleration sensed yields [1]:

$a_{n}=\left(\left(l_{p} M_{\alpha}-Z_{\alpha}\right) \alpha+\left(l_{p} M_{q}-Z_{q}\right) q+\left(l_{p} M_{\eta}-Z_{\eta}\right) \eta\right) / 9.81 \quad[\mathrm{~g}]$

From transforming state-space model into transfer function [8], the numerator of forward-path transfer function equals:

$$
\begin{aligned}
N\left(l_{p}\right) & =-60\left(l_{p} M_{\eta}-Z_{\eta}\right) s^{2}+\left(M_{w} Z_{\eta} l_{p}-M_{\eta} Z_{w} l_{p}+M_{q} Z_{\eta}-M_{\eta} Z_{q}\right) s+M_{w} Z_{\eta} u_{0}-M_{\eta} Z_{w} u_{0} \\
& =\left(2.922 l_{p}-5.162\right) s^{2}+\left(0.3825+0.3788 l_{p}\right) s+146.7
\end{aligned}
$$

Table 3 shows the elevator-to-normal-acceleration transfer function zeros for a range of accelerometer positions from the c.g. forward with distance $1_{\mathrm{p}}$.

Table 3 shows that as the accelerometer position is moved forward, the non-minimum phase zero moves out toward infinity, thus keeping constant the transfer function dc gain. Eventually, the outer loop sensitivity changes sign and a zero comes in from infinity along the negative real axis, finally combining with the other real zero to form a complex pair $[1,5,10,11]$. 
Table 3 Change of zeros with accelerometer position

\begin{tabular}{l|l}
\hline \hline $\mathrm{lp}[\mathrm{m}]$ & Forward-path transfer function numerator \\
\hline 0 & $(-5.162)(s-5.368)(s+5.294)$ \\
\hline 0.5 & $(-3.701)(s-6.3736)(s+6.219)$ \\
\hline 1 & $(-2.24)(s-8.2643)(s+7.9245)$ \\
\hline 1.5 & $(-0.779)(s-14.347)(s+13.126)$ \\
\hline 1.7 & $(-0.1946)(s-30.22)(s+24.945)$ \\
\hline 1.9 & $(0.3898)(s+1.414-19.35 j)(s+1.414+19.35 j)$ \\
\hline \hline
\end{tabular}

\section{Choice of Accelerometer Position}

From Table 3 at a position near 1.9 [m] forward of the c.g. the non-minimum phase effect disappears, and this point corresponds to an "instantaneous center of rotation" when an elevator input is suddenly applied. If the accelerometer is placed at this location, the system poles and zeros will be completely located in the left hand plane and then it will not be hard for the system to be compensated. It is also important to place the accelerometer close to a node of the most important fuselage bending mode. If this is not done, structural oscillations will be coupled into the rigid-body control system and may degrade the flying qualities $[1,2,3,11]$.

It is necessary at first to find the position of the instantaneous center of rotation. The numerator of the forward-path transfer function is:

$$
\begin{aligned}
N\left(l_{p}\right) & =C^{\prime}\left[s I-A^{\prime}\right]^{-1} B^{\prime} \\
& =\left(l_{p} M_{\eta}-Z_{\eta}\right) s^{2}+\left(M_{\eta} Z_{w} l_{p}-M_{w} Z_{\eta} l_{p}+M_{q} Z_{\eta}-M_{\eta} Z_{q}\right) s+M_{w} Z_{\eta} u_{0}-M_{\eta} Z_{w} u_{0}
\end{aligned}
$$

Dividing the whole equation by $\mathrm{s}^{2}$ and then finding the limit at $\mathrm{s} \rightarrow \infty$ yields:

$$
\begin{aligned}
& \lim _{s \rightarrow \infty}\left[\left(l_{p} M_{\eta}-Z_{\eta}\right)+\frac{\left(M_{\eta} Z_{w} l_{p}-M_{w} Z_{\eta} l_{p}+M_{q} Z_{\eta}-M_{\eta} Z_{q}\right)}{s}+\frac{M_{w} Z_{\eta} u_{0}-M_{\eta} Z_{w} u_{0}}{s^{2}}\right]=0 \\
& \Rightarrow\left(l_{p} M_{\eta}-Z_{\eta}\right)=0
\end{aligned}
$$

Finally the value of $1_{p}$ at which the non-minimum phase zero moves towards infinity is:

$$
l_{p \min }=\frac{Z_{\eta}}{M_{\eta}}
$$

where $1_{p}<1_{p \min }$ yields to locate the non-minimum phase zero in the right hand plane while $1_{p}>$ $1_{\text {pmin }}$ moves the non-minimum phase zero to the left hand plane combining with the other real zero to form a complex pair.

However, it must be taken into consideration that missile c.g. changes its position during powered phase while the value of $Z_{\eta}$ and $M_{\eta}$ changes during the flight time. This means that $l_{p m i n}$ changes during the whole flight time whereas the accelerometer position is fixed during flight 
time and it is very hard to move it along the missile longitudinal axis during flight. To overcome this problem, the value of $1_{\text {pmin }}$ would be calculated at the whole design points and at every point the distance from missile nose to accelerometer $\left(\mathrm{x}_{\mathrm{a}}\right)$ is calculated and then the minimum value will be chosen to be the accelerometer position as shown in Table 4 . The minimum value of this distance guarantees that the whole zeros of the forward path transfer function are always placed in the left hand plane during the whole flight even they were real or imaginary.

Table 4 The value of $l_{p m i n}$ and accelerometer position $x_{a}$ at design points

\begin{tabular}{c|c|c|c|c|c|c}
\hline \hline Point & Time $[\mathrm{sec}]$ & $\mathrm{Z}_{\eta}$ & $\mathrm{M}_{\eta}$ & $\mathrm{l}_{\mathrm{pmin}}[\mathrm{m}]$ & $\mathrm{x}_{\mathrm{cg}}[\mathrm{m}]$ & $\begin{array}{l}\mathrm{x}_{\mathrm{a}}[\mathrm{m}] \\
=\mathrm{x}_{\mathrm{cg}}-1_{\mathrm{pmin}}\end{array}$ \\
\hline 1 & 1 & -0.0211 & -0.0128 & 1.648438 & 4.89 & 3.241563 \\
\hline 2 & 5 & -0.8459 & -0.4778 & 1.770406 & 4.804 & 3.033594 \\
\hline 3 & 10 & -1.7718 & -0.9234 & 1.918778 & 4.652 & 2.733222 \\
\hline 4 & 13.05 & -2.1708 & -1.04 & 2.087308 & 4.531 & 2.443692 \\
\hline 5 & 20 & -1.1815 & -0.5718 & 2.066282 & 4.441 & 2.374718 \\
\hline 6 & 40 & -0.1313 & -0.0646 & 2.032508 & 4.438 & 2.405492 \\
\hline 7 & 90 & -0.0141 & -0.0069 & 2.043478 & 4.438 & 2.394522 \\
\hline 8 & 150 & -0.2595 & -0.1279 & 2.028929 & 4.438 & 2.409071 \\
\hline 9 & 180 & -2.5379 & -1.248 & 2.033574 & 4.438 & 2.404426 \\
\hline \hline
\end{tabular}

Then $x_{a}=x_{a m i n}$ along the flight time and hence $x_{a}=2.35[\mathrm{~m}]$ and this position guarantees the elimination of the non-minimum phase behavior of the normal acceleration response along flight time.

\section{Conclusion}

The optimum position for acceleration sensor in surface-to-surface aerodynamically tailcontrolled missile is determined. The mathematical model was derived and the aerodynamic coefficients for this model were calculated using Missile Datcom. The inner loop of the normal acceleration autopilot is designed to increase the damping ratio of the open-loop poles of the outer loop. Gain scheduling is used to maintain the value of damping ratio along flight time. The open-loop zeros are calculated in case of moving accelerometer ahead of missile c.g. till they become imaginary. The position of accelerometer which eliminates non-minimum phase behavior is determined at specific point. The position of accelerometer is then calculated along flight time and the most suitable is chosen.

\section{References}

[1] Brian L. Stevens, Frank L. Lewis, "Aircraft Control and Simulation”, John Wiley \& Sons, Inc., (1992).

[2] Abdelbaset El Ramlawy, "Multivariable flight control systems for agile combat aircraft", University of Salford, PhD thesis, 1992. 
[3] Ridgely, D.B., Banda, S.S., "Introduction to Robust Multivariable Control" AFWAL-TR85-3102, Flight Dynamics Laboratory, Air Force Wright Aeronautical Laboratories, Dayton, Ohio, February, 1986.

[4] John H. Blacklock, "Automatic Control of Aircraft and Missile", 2nd edition, John Wiley \& Sons, Inc., (1991).

[5] William B. Blake," Missile DATCOM, User's Manual -1997 FORTRAN 90 Revision". AFRL-VA-WP-TR-1998-3009, Feb. (1998).

[6] M. S. Mohamed, A. M. Bayoumy, A. A. El-Ramlawy, G. A. El-Sheikh, " Comparison of Classical and Predictive Autopilot Design for Tactical Missile", ASAT - 15 - Military Technical College, Cairo, EGYPT, May 28 - 30, 2013.

[7] Farhan A. Faruqi , Thanh Lan Vu," Mathematical Models for a Missile Autopilot Design", Weapons Systems Division,Systems Sciences Laboratory,DSTO-TN-0449,(2002).

[8] Benjamin C. Kuo, Farid Golnaraghi, "Automatic Control Systems”, 9th ed., John Wiley \& Sons, Inc., (2010).

[9] Karl Johan Aström, "Adaptive control”, 2nd ed., Addison Wesley Publishing Company, 1995.

[10] D. Mcruer, I. Ashkenas, D. Graham, “Aircraft dynamics and automatic control”, Princeton University Press, (1973).

[11] Mostafa S. Mohamed, "Autopilot Design for Ballistic Missiles", Military Technical College, M.Sc. thesis, 2013. 